\title{
Del conflicto a la cultura de paz: implicaciones
}

\section{Sociedad del conocimiento}

La sociedad actual en la que los grandes avances de la humanidad están ganando la batalla tecnológica: conquista del espacio; investigación sobre el genoma humano... etc., la llamada "Sociedad del conocimiento" (Drucker, 1969) se enfrentan al reto de proyectarse y adaptarse a un proceso de cambio que viene avanzando muy rápidamente hacia la construcción de Sociedades del Conocimiento.

Del mismo modo surge la sociedad de la información, como instrumento del conocimiento, que con el incremento de las transferencias de información modifica en muchos sentidos, la forma en que se desarrollan muchas actividades de la sociedad moderna.

La Declaración de Principios de Ginebra (CMSI, 2003,a), expresa en su primer artículo: "Nosotros declaramos nuestro deseo y compromiso comunes de construir una sociedad de la información centrada en la persona, integradora y orientada al desarrollo..."

Aún hoy, y a pesar de todo, los humanos enfrentamos la gran paradoja de la incomunicación dentro de la sociedad de la comunicación, siendo así que nos encontramos en multitud de ocasiones en que utilizando las tecnologías puntas en comunicación carecemos de "comunicación" en el sentido más profundo de las relaciones del ser humano.

Pasamos, miramos, pero a veces no vemos, el acto de cognición implica un proceso por el que percibimos, tomamos conciencia de lo percibido, lo incorporamos y finalmente hacemos uso de ello. Dicho proceso precisa de un elemento esencial, la conciencia del ser. Es la conciencia de lo que hacemos lo que racionaliza nuestros actos.

El conocimiento obedece a aquellos elementos que pueden ser comprendidos por la mente humana razonable. Para ello el acto de aprender requiere la voluntad del sujeto, amen de la implicación de sus aptitudes y actitudes, es decir, en el acto de aprender se implica el individuo integralmente.

Tal y como demuestran las investigaciones sobre la inteligencia múltiple y los estudios que demuestran la importancia de la inteligencia emocional para la cognición, es necesario que repensemos la forma de aprender y la importancia que tiene en el aprendizaje la "inteligencia emocional". Es necesario

\section{Revista Iberoamericana de Educación}

ISSN: 1681-5653

n. ${ }^{\circ} 44 / 5$ - 25 de noviembre de 2007

EDITA: Organización de Estados Iberoamericanos

para la Educación, la Ciencia y la Cultura (OEI) 
contemplar este dato dada la importancia de las emociones en las vidas de cada individuo: "Se produce una interacción permanente entre emoción comportamiento y pensamiento de tal forma que se retroalimentan permanentemente" (Bisquerra, Internet).

En el campo de la educación, el informe Delors perfiló el camino hacia una sociedad del siglo XXI en la que la educación debe plantearse como meta una sociedad para todos, habla de una "utopía necesaria" en la cual toda persona tenga la oportunidad de desarrollarse como "única".

Se refuerza en dicho informe la importancia del conocimiento, entendiéndolo como una cognición que conduce al desarrollo de todas las capacidades, competencias necesarias y útiles para convivir, para hacere implicarse en nuestro contexto, en definitiva para ser persona o lo que es lo mismo ciudadana activa en la sociedad.

\section{ELEMENTOS ESENCIALES DEL INFORME DELORS}

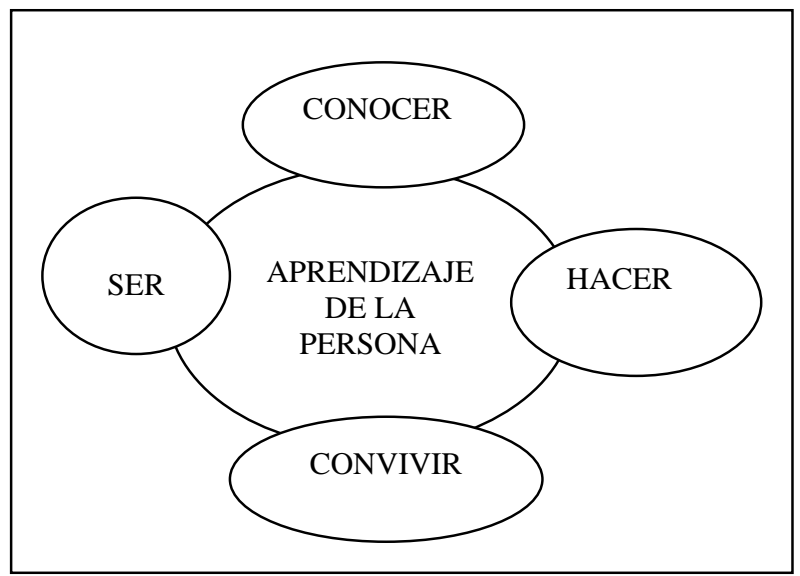

En el desarrollo de habilidades-competencias queremos destacar la importancia de la comunicación, como habilidad que permite y facilita el intercambio y la interacción entre las personas y los grupos.

En opinión de Monjas, M. I. (1999, p. 28), las habilidades sociales son las "conductas o destrezas sociales especificas requeridas para ejecutar competentemente una tarea de indole interpersonal. Implica un conjunto de comportamientos adquiridos y aprendidos y no un rasgo de personalidad. Son un conjunto de comportamientos interpersonales complejos que se ponen en juego en la interacción con otras personas".

Podemos decir que hablar de comportamientos es hablar asímismo de motivos últimos de la persona que se fundamentan en valores y creencias. Es como hablar de la cultura imperante en su grupo o contexto. Es decir, que en las relaciones humanas encontramos, como telón de fondo, un bagaje de concepciones y actifudes que determinan diferentes posiciones ante las situaciones que se producen en cada momento y espacio contextual.

En esta línea de pensamiento es obvio que no siempre los intereses de las personas, los integrantes de cada grupo o sociedad estén de acuerdo. Ante el desacuerdo surge el conflicto. 


\section{Diversidad, diferencia, ¿conflicto?}

Con el término "Diversidad" nos introducimos en el mundo de las diferencias y en palabras de Gimeno, en un mundo de ambigüedad, porque según este autor "diversidad alude a la circunstancia de ser distinto y diferente, pero también a la de ser desigual" (Gimeno, 1995, p. 20).

En realidad, el término de diversidad evoca un pensamiento complejo y a veces utilizamos términos para definir la diversidad que acaban refiriéndose a las diferencias.

Queremos reseñar que la "diversidad y las diferencias" son un hecho constatado e ineludible en la naturaleza. Sin embargo, las desigualdades que derivan sobremanera de la diversidad y de las diferencias son hechos creados y provocados por la estructura social.

Hablar de diversidad en el campo de la educación (utópica), es hablar de educación para todos sin ningún tipo de excepción. Atrás parecen quedar las concepciones de "educación especial" y con ellas la idea de educación segregadora cuando el discente presentaba alguna diferencia en su ser o en su contexto.

Podemos decir que el fundamento filosófico que impulsa el movimiento de "educación en la diversidad", parte para su desarrollo de un supuesto básico que es el valor del ser humano, en sí mismo, lo cual trasladado al ámbito social, en el que se desarrolla, supone el reconocimiento de los derechos plenos de todos los ciudadanos (en el derecho positivo de cada país), lo que implica por parte de los distintos estados, la articulación de las medidas necesarias para que las oportunidades sean reales para todos.

Siendo así que el concepto de educación y su dimensión personal trasciende a lo social, dado que la educación es una actividad eminentemente social y reconocida como derecho ineludible en la legislación internacional.

Y en el marco supranacional, es un hecho innegable que cada sociedad, con su propia cultura, forma parte de una sociedad más amplia: el mundo. Una "aldea global", universal, que en la actualidad esta regida por una normativa común basada en unos principios y valores para todos sus componentes. Tal extremo fue reconocido en la Declaración de los Derechos Humanos, adoptada por la O.N.U. en París en el año 1948. Según Cotta, S. (1990), citado en Medina (1998), este acontecimiento sucedió como reconocimiento a la revolución "jurídico política", que se manifiesta en la sociedad desde mediados del siglo $X X$, y que consigue dotar a la comunidad internacional de un "conjunto de principios normativos ético-políticos y educativos fundamentales de validez para todos los hombres". (Medina, 1998, p. 64). A partir de esta proclamación "los Derechos Humanos son unos derechos inherentes e inexcusables a la condición humana que deben ser reconocidos, declarados y garantizados, por leyes positivas" (Medina, 1998, p. 66).

Actualmente, el conflicto puede considerarse como un rasgo inevitable de las relaciones sociales, que en esencia no tiene por qué ser negativo o perverso. La dificultad estriba en que todo conflicto puede adoptar un curso constructivo o destructivo y por lo tanto, la cuestión no es tanto eliminar o prevenir el conflicto, sino saber asumir dichas situaciones conflictivas y enfrentarse a ellas con los recursos suficientes para que todos los implicados en dichas situaciones puedan salir enriquecidos. No se trata de que existan ganadores o perdedores sino de construir una cultura basada en la colaboración y el acuerdo. 
Para definir escuetamente los rasgos de un conflicto partimos de la definición siguiente: "Divergencias percibidas de intereses, o una creencia de que las aspiraciones actuales de las partes no pueden ser alcanzadas simultáneamente"(Rubin, Pruitt y Hee Kim, 1994).

En esencia, el conflicto es legitimo desde el punto de vista de la singularidad, siempre que la solución adoptada se fundamente en el respeto, la justicia, la equidad, etc., valores todos ellos básicos y fundamentales en la construcción de una ciudadanía humanista y democrática.

De esta forma, teniendo en cuenta a las partes del conflicto y sus intereses, podría llegarse a la situación de prevenir, en el sentido de adoptar otro tipo de conductas no conflictivas, en las que las parte puedan considerarse satisfechas y en cuyo caso no existiría ni ganador ni perdedor.

Siendo que el comportamiento se fundamenta en motivo fundamentados, en creencias y valores, es necesario repensar la educación en valores universalmente válidos que se anclan en los Derechos Humanos y en las conquistas de las leyes positivas de la sociedad actual, concretadas en cada país o contexto.

Todo ello en el fundamento de valores irrenunciables para una vida digna:

a) Dignidad humana: "Es el valor básico o referente principal de las necesidades que la persona tiene en la esfera moral o jurídica" (Medina Rubio, 1998, p. 83). Este valor, sagrado, incluye el derecho de toda persona a la diferencia, y a la igualdad.

b) Libertad: "Es un valor supuesto o derivado del derecho de la dignidad humana". La libertad es la que otorga al individuo el rango de "fin en sí mismo", pero la libertad adquiere tantas dimensiones como vertientes en las que puede ser entendida: como facultad de determinación, o ausencia de coacciones; como capacidad de decidir por sí mismo; como dimensión comunitaria...

c) Autonomía y responsabilidad: "Autonomía y responsabilidad implican participación activa y libre en la realización de los valores" (Medina, 1998, p. 85) Se trata de un valor relacional, estando ambos en conexión con el resto de los valores acatados por el individuo, ya que supone una respuesta personal y reflexiva ante las decisiones.

d) Igualdad y derecho a la diferencia personal: Es un valor que emana del derecho referido a la igualdad básica, tanto en derechos como en oportunidades. Valor que resulta ineludible en educación, en cuanto no sólo se trata del derecho de toda persona a la educación, sino la obligación que de este derecho se deriva para los gobiernos, quedando obligados a proporcionar "oportunidades" educativas.

O valores considerados como Ethos democráticos en las relaciones humanas cuales son:

a) Tolerancia: "Como principio que proclama la igualdad de todos los hombres, que tienen como una de sus barreras el rechazo y la intolerancia hacia los que les incomodan" (Medina Rubio, 1998, p. 85). Generalmente este valor supone e implica otros valores como son: apertura hacia el otro, sentido de la generosidad, relaciones sustentadas en la veracidad, fidelidad, paciencia y tenacidad. 
b) Justicia: Como "virtud general que dirige o pone en ejercicio los actos de las demás virtudes, ya que la justicia incluye, de alguna manera, todos los valores anteriormente citados". (Medina Rubio, 1998, p. 86).

c) Solidaridad: Es un valor que nos conduce a tener en cuenta a los demás, a estar vinculados con los demás como parte activa. En este sentido compromete y crea actitudes de participación con los demás.

d) Paz: Es un valor que requiere eliminar formas de violencia, crear formas de participación activa en el proceso humano para hacerlo más agradable a los participantes. La paz no es una conquista, la paz es siempre un camino.

e) Coraje cívico: "Como tenacidad, fortaleza de ánimo en la fidelidad a las propias convicciones" (Medina Rubio, 1998).

f) Diálogo: "Actitud dialógica que requiere atenerse a las condiciones de un diálogo efectivo: la disposición al razonamiento, a la crítica y a la argumentación, la exposición razonada de motivos, la capacitación para discutir y lograr acuerdos, reconocimiento de interlocutores válidos; capacidad de empatía, actitud comunicativa, capacidad de hacerse cargo de las consecuencias de las acciones, disponibilidad y generosidad". (Medina, 1998, p. 87).

Tales valores posibilitan a las partes una concepción justa, equitativa y equilibrada de las divergencias existentes.

\section{Abordar el conflicto desde una cultura diferente. La cultura de paz. Implicaciones}

Cambiar la resolución, cambiar el conflicto. Negociación

Con este bagaje de pensamiento y con valores fundamentados en la esencia y la riqueza de la singularidad de la persona el abordaje del conflicto cambia.

\section{En todo conflicto existen partes}

1) Las partes. Todo conflicto está integrado por partes, ya hablemos de personas, grupos u organizaciones más o menos extensas. Son por tanto, las partes integrantes del conflicto las que presentarán intereses y puntos de vista de acuerdo con sus objetivos y valoraciones. Al considerar las partes, es posible que éstas existan con más o menos importancia. Podemos hablar pues de partes:

a) Principales, tienen un interés directo en el conflicto y persiguen sus metas activamente para promover sus propios intereses.

b) Secundarias, tienen interés en el resultado de un evento pero pueden o no percibir que hay conflicto o decidir o no si juegan un papel activo en el proceso de toma de decisiones.

c) Intermediarias, generalmente intervienen para facilitar la resolución del conflicto y mejorar la relación entre las partes. 


\section{En todo conflicto existe un asunto y unos objetivos de las partes}

El asunto pues nos introduce en el componente y en la esencia del conflicto. A su vez el componente del conflicto estará sustentado por los intereses y valores de las partes que lo componen. En tal sentido podemos contemplar:

a) Los intereses de las partes; son los acuerdos de las partes sobre determinadas posiciones, roles o recursos.

b) Los valores. Son las percepciones de las partes sobre lo deseable.

En cuanto a los objetivos, si bien son los que definen la esencia del conflicto, es cierto que habremos de delimitar diferentes tipos:

c) Positivos, reflejan futuros resultados tangibles, como "seguridad de las fronteras" o "estado independiente".

d) Negativos, reflejan el interés de evitar un resultado no deseado.

\section{Finalmente hemos de hablar de diferentes tipos y niveles de conflictos}

De acuerdo con el planteamiento de Moore (1995), puede establecerse una clasificación en función de las partes implicadas o las causas del conflicto que tendría la siguiente descripción:

- Los conflictos de relación, se deben a fuertes emociones negativas, percepciones falsas o estereotipos, a escasa o nula comunicación, o a conductas negativas repetitivas.

- Conflictos de información, se dan cuando no existe la información necesaria para que las partes adopten decisiones correctas.

- Conflictos de intereses, se ocasiona por la competición entre necesidades incompatibles o percibidas como tales.

- Conflictos estructurales, son causados por estructuras opresivas de las relaciones humanas, que pueden ser incluso consecuencia de fuerzas externas a las partes del conflicto.

- Conflictos de valores, son causados por sistemas de creencias incompatibles.

Existen clasificaciones basadas en los niveles del conflicto, en el que predomina el criterio de los actores o personas implicadas. Desde esta perspectiva los conflictos pueden ser: intrapersonales, interpersonales, intragrupales e intergrupales (Lewiski, Litterer, Minton y Saunders, 1994).

- Conflicto intrapersonal o intrapsíquico: ocurre dentro de los individuos.

- Conflicto interpersonal: ocurre entre personas individuales y dentro de la convivencia familiar, laboral o de amigos. 
- Conflicto intragrupal, se suele dar en pequeños grupos.

- Conflicto intergrupal, se produce entre dos grupos constituidos socialmente, naciones, sindicatos, etc.

\section{La resolución del conflicto. Cambiar para vivir con paz}

Los desacuerdos, problemas y conflictos pueden surgir, prácticamente, en cualquier relación. Normalmente afrontamos dichas situaciones de manera informal. Podemos observar que en la vida diaria, la mayor parte de nuestros desacuerdos se solucionan mediante este procedimiento, o bien logrando acuerdos que satisfacen más o menos, a las distintas partes implicadas, o bien abandonando el asunto porque carece de interés o no tiene solución.

\section{La negociación}

Además de las conversaciones informales existe otro procedimiento para alcanzar acuerdos mutuamente satisfactorios, es la negociación. Por medio de la negociación las distintas partes que perciben que tienen conflictos de intereses tratan de llegar a acuerdos que beneficien sus pretensiones. Para ello se reúnen para informarse mutuamente de sus necesidades e intereses, intercambiar recursos y posibles soluciones.

Las actividades de negociación han de contemplar:

1) Planificar y analizar. Sobre el conflicto, implicados, soluciones, partes. Ello implica realizar un mapa del conflicto en el que se realice un análisis sobre los motivos, problemas, discrepancias, intereses, barreras, diferencias de valores, obstáculos.

2) Establecer un tono positivo, creación de un proceso negociador con voluntad de hallar soluciones viables. Reconocer y validar a la otra persona.

3) Discutir y definir el problema. Crear procesos menos costosos. Identificar posturas y obstáculos.

4) Generar ideas y soluciones. Ordenar los procedimientos de resolución de conflictos, en orden a conseguir el máximo beneficio con el mínimo coste.

5) Evaluar las soluciones. Para poder medir el alcance de cada parte en cuanto a los objetivos alcanzados y las concesiones realizadas. Determinar por tanto las ventajas y desventajas para cada curso de acción y hacer "agente de la realidad".

6) Decidir un curso de acción.

\section{El tercer lado}

Cuando aún no se puede llevar a cabo el proceso negociador debido a que las partes no se ponen de acuerdo, se necesita ayuda de alguien ajeno al conflicto, es el caso de la mediación. La medicación 
implica la intervención de una tercera parte, aceptable por todos, que no tiene autoridad para tomar decisiones.

El tercer lado es una forma de ver los Conflictos que nos rodean no sólo desde un lado, o el otro, sino desde una más amplia perspectiva: la de la comunidad que lo circunda.

Según Stenhouse (1997, p. 15): "Las afirmación de las diferencias no pueden olvidar la existencia de importantes aspectos comunes en la experiencia humana más diversificada". Hay que abrir caminos a lo común, los seres humanos aunque individual y socialmente distintos compartimos experiencias comunes a todos.

En el proceso de resolución del conflicto, cuando contravalores como temor, rabia o resentimiento se apoderan del conflicto su proceso entra en una escala de violencia y de falta de control. No obstante si el camino escogido contempla valores como amor, justicia, objetividad, equidad, etc, todo ello opera para moderar el conflicto.

El tercer lado en su intervención debe buscar que el conflicto entre en fase donde el valor de la razón y la emoción en mente estén controladas.

El tercer lado en su papel de interventor del conflicto, como un tercero neutral, debe identificar los intereses reales de los actores en conflicto y lograr caminos de encuentro, donde inclusive las metas incompatibles puedan dar lugar a beneficios compatibles.

\section{Qué es la cultura de paz}

Resulta innegable que la violencia (directa, estructural, cultural y/o simbólica) está presente y puede observarse en todos los rincones de nuestro planeta. Es por ello que urge afanarse en aportar cada uno y cada cual el componente necesario para la construcción de la paz.

La educación para la paz pretende alcanzar la construcción de un nuevo mundo (orden internacional), basado en un concepto de paz. Paz que traspasando la interculturalidad se constituya en ecuménica, atravesando las fronteras de las diferencias de las culturas busque los aspectos comunes que existen entre ellas y hallen el patrón universal que las identifica.

Sólo de este modo las personas, los pueblos y las naciones poseen el futuro y con él la posibilidad de corregir su rumbo a través de una educación para la paz. Y ello contemplado en cualquier nivel (individual, familiar, social, nacional, internacional) tiene como resultado la solución no violenta de los conflictos y la justicia.

\section{Fundamentos de la práctica para una cultura de paz}

La paz debe fundamentarse en la práctica de los derechos humanos, que representa una toma de partido en el proceso de socialización, por valores que alimenten el cambio social y personal. 


\section{Los Derechos Humanos como garantes de valores imprescindibles para la paz: La carta de la tierra}

\section{La paz y sus dimensiones}

Estamos de acuerdo en el planteamiento realizado por Fernández Herrerías (2004), en el que plantea diferentes dimensiones de la paz.

1. Paz social

Es la dimensión social de la paz, que junto con la dimensión ecológica y la dimensión interna conforman una nueva dimensión de paces distinguibles pero no separadas de una concepción integral de paz (Fernández Herrería, 2004).

Es una dimensión de la paz que tiene en cuenta el proceso de desarrollo sustentable de los seres humanos y de los pueblos, desarrollo definido no sólo por los Derechos Humanos de segunda generación (sociales, políticos, económicos y culturales, derechos civiles, etc.), sino también en los de la tercera generación (solidaridad, derecho a la paz, al desarrollo, a un medio ambiente sano y equilibrado), y en la cuarta generación de derechos (bioética).

Es un concepto de paz que piensa que el desarrollo debe ser para todos los seres que habitan el planeta.

Este concepto de paz se enmarca en la corriente de las grandes conferencias, ONU a partir de 1992, Berlín 1996, Kyoto 1997, Buenos Aires 1998, La Haya, 2000. Además de todo un conjunto de conferencias mundiales de Derechos Humanos. Viena 1993, El Cairo, 1994, Copenhague, 1995, Beiijn, 1995. Turquía 1996.

\section{Paz Gaia}

Es la dimensión ecológica o natural de la paz. Dado que no es posible separar las tres dimensiones de la paz, no será posible una paz global, es decir un desarrollo humano sostenible para toda la humanidad sin respetar también los derechos del medio ambiente.

La idea de una paz gaia surge de la idea de una ecología como paradigma, basado en una nueva inteligibilidad sistémica y compleja donde se niega la fragmentación de los seres humanos entre sí y su aislamiento del entorno y del observador. Vivimos fragmentados internamente y separados de nosotros mismos. Somos seres humanos cosificados y perdidos. La fragmentación es la nueva forma en que se presenta la violencia cultural.

El viejo esquema darwiniano de la supervivencia del mas apto, tiende a sustituirse por el esquema ecológico de la supervivencia del más cooperativo" (Paniker, 1984, p. 28).

La hipótesis gaia, formulada en un principio por Lovelock (1979) y completada por Margulis (1989) es en realidad una nueva teoría de la evolución de las especies con la evolución de su entorno natural, es decir, da una perspectiva ecológica a la teoría de la evolución que hace conveger en un único proceso la intuición 
de Charles Darwin de la evolución de las especies y de la evolución del entorno. Lovelock escribe al principio de su obra lo siguiente:

El conjunto de los seres vivos de la tierra [...] puede ser considerado como una entidad viviente capaz de transformar la atmósfera del planeta para adecuarla a sus necesidades globales" (Lovelock, 1983, p. 22).

\section{Paz interna}

Dice el preámbulo de la Declaración de los Derechos Humanos: "Si las guerras nacen en la mente de los seres humanos, es en la mente de los seres humanos donde deben erigirse los baluartes de la paz".

La paz tiene tres dimensiones, o ámbitos de expresión: paz de los seres humanos entre si (sociall; la paz de los seres humanos con la naturaleza (dimensión natural o ecológica de la paz); y la paz de los seres humanos consigo mismo (dimensión interna).

Según Fernández Herrerías nuestra cultura occidental volcada más hacia lo externo ha mostrado en general las siguientes posiciones ante este tema:

1) No reconocer de hecho, las dimensiones transpersonales del desarrollo humano.

2) Cuando se reconoce esa dimensión interna o al menos se recogen esas tradiciones culturales que asi lo viven, se asume también una dimensión espiritual de la paz, aunque se considera sólo en el ámbito de lo privado y como una paz intimista, circunscrita al sujeto.

3) Relacionado con la postura anterior, se tiende a separar lo exterior de lo interior, las elaboraciones de la paz occidentales, de las orientales (y de otros pueblos) como si lo social político, económico y ecológico estuviera separado de lo interior, de la tendencia hacia la autorrealización y la trascendencia.

- El mundo es lo que nosotros somos, de ahí que el problema del individuo es el problema del mundo (Krishnamurti).

- Tiene que darse una revolución fundamental empezando por el individuo ya que la paz de la persona es la paz del mundo (Krishnamurti).

\section{Conclusiones}

1) Reflexión en torno a la escalada de violencia de manera directa, estructural, cultural o simbólica.

2) Destacar la importancia de la educación en valores como requisito para formar ciudadanos activos, democráticos y comprometidos en la configuración de un mundo habitable, sostenible y para todos.

3) Importancia de la resolución positiva del conflicto con beneficio para todos, negociando, tomando decisiones para el acuerdo, llegando a aquello que nos une más que a lo que nos separa. 
4) Resaltar la importancia de la Paz, fundamentada en la Paz Social, la Paz Ecológica y la Paz Personal e interna.

Con todo ello: Nada de lo humano nos puede resultar indiferente y ajeno.

\section{Bibliografía}

BELMONTE NIETO, M. (1998): Atención a la diversidad. Bilbao: Mensajeros.

CASAMAYOR, G., y otros (1999): Cómo dar respuesta a los conflictos. Barcelona: Graó.

CHICA JIMÉNEZ, M. (2005): Tutoría y atención a la diversidad en Educación Secundaria (Estudio de casos). Tesis inédita. Universidad de Sevilla.

DECLARACIÓN DE PRINCIPIOS DE GINEBRA (2003): Construir la sociedad de la información: un desafio global para el nuevo milenio. http://www.itu.int/wsis/docs/geneva/official/dop-es.html [abril de 2007]

DRUKER, P. (1969): The Age of Discontinuity: Guidelines in our Changuen Society. New York.

FERNANDEZ, HERRERÍA, ALFONSO, et al. (1994): Paz y prospectiva: problemas globales y futuro de la humanidad. Granada: Universidad de Granada.

GIMENO SACRISTÁN (1995): Diversos y también desiguales. ¡Qué hacer en educacióni Kikiriki, 38, pp. 18-38.

HARGREAVES, Andy (1998): "Paradojas del cambio: la renovación de la escuela en la era Postmoderna", en Kikiriki, 49, pp. 16-24.

INFORME DELORS (1996): La educación encierra un tesoro. Madrid: Santillana-UNESCO.

LOVELOCK, J. (1983): Gaia: Una nueva visión de la vida sobre la Tierra. Hermann Blume Editores.

MEDINA RUBIO, R. (1998): "Los derechos humanos y la educación en los valores de una ciudadanía universal", en Aula abierta, 72, pp. 63-95.

MONJAS, M. I. (1999): Programa de enseñanza de habilidades de interacción social para niños y niñas en edad escolar. Madrid: CEPE.

MOORE, C. (1995): El proceso de mediación. Barcelona: Granica.

RODRIGUE ESTRADA, M. (1989): Manejo de conflictos. México: El Manual Moderno.

STENHOUSE, L. (1997): Ideología, pensamiento y educación. Morón, Sevilla: Kikiriki. Cooperación andaluza. 